\title{
Circular RNAs Serve as Prognostic, Diagnostic and Clinicopathological Markers in Lung Cancer: An Updated Meta-Analysis
}

\section{CURRENT STATUS: UNDER REVISION}

BMC Cancer $\triangle \mathrm{BMC}$ series

Zhuozheng Liang

Third Affiliated Hospital of Sun Yat-Sen University

Cui Chen

Guangdong Medical University

Cheng Guo

Third Affiliated Hospital of Sun Yat-Sen University Department of Otorhinolaryngology Head and Neck Surgery

Ping Meng

Third Affiliated Hospital of Sun Yat-Sen University

Manman Zou

Third Affiliated Hospital of Sun Yat-Sen University

Liyi Zhang

Third Affiliated Hospital of Sun Yat-Sen University

Hui Liu

Third Affiliated Hospital of Sun Yat-Sen University

Tiantuo Zhang

Third Affiliated Hospital of Sun Yat-Sen University

\itcinmatalzz@163.comCorresponding Author

ORCiD: https://orcid.org/0000-0001-7496-8135

\section{DOI:}

$10.21203 / \mathrm{rs} .2 .24261 / \mathrm{v} 1$

\section{SUBJECT AREAS}

Cancer Biology Oncology

\section{KEYWORDS}

Lung cancer, Circular RNA, Diagnosis, Prognosis, Meta-analysis 
Abstract

In recent years, the roles of circular RNAs (circRNAs) in the biogenesis and clinical application have gradually garnered much interest in the field of cancer research. However, high-quality studies evaluating the roles of circRNAs in clinicopathological feature and clinical application of lung cancer are still unrevealed. Herein, we aimed to elucidate the functions of circRNAs in the association with prognosis, diagnosis and clinicopathology in lung cancer. Comprehensive and reasonable search strategies were used in four databases up to Nov. 24th 2019. A total of 50 studies with 3815 patients were incorporated into our meta-analysis, in which 42 focused on clinical characteristics, 10 related with diagnosis (16 items were eligible), and 31 were aimed at prognosis. In terms of clinical characteristics, both odds ratio (OR) and 95\% confidence interval $(95 \% \mathrm{Cl})$ were shown that circRNAs were significantly associated with TNM, tumor differentiation, lymph node metastasis, distal metastasis and tumor size in lung cancer. For diagnosis, both upregulated and downregulated circRNAs distinguished patients with lung cancer from healthy people with pooled sensitivity (Sen), specificity (Spe) and area under curve (AUC), of which the values were $0.78,0.76$ and 0.81 respectively. In the section of prognosis, circRNAs can better predict the survival time of patients with lung cancer (overall survival, OS: $\mathrm{HR}=0.46,95 \% \mathrm{Cl}: 0.33-0.65$ in downregulated circRNAs; $\mathrm{HR}=2.23$, $95 \% \mathrm{Cl}: 1.97-2.53$ in upregulated circRNAs). In summary, the results presented here suggested that circRNAs were the potent cancer biomarkers in clinicopathology, diagnosis and prognosis of lung cancer patients, which deserve further application in clinical practice.

Full Text

Due to technical limitations, full-text HTML conversion of this manuscript could not be completed. However, the manuscript can be downloaded and accessed as a PDF.

Tables

Figures 


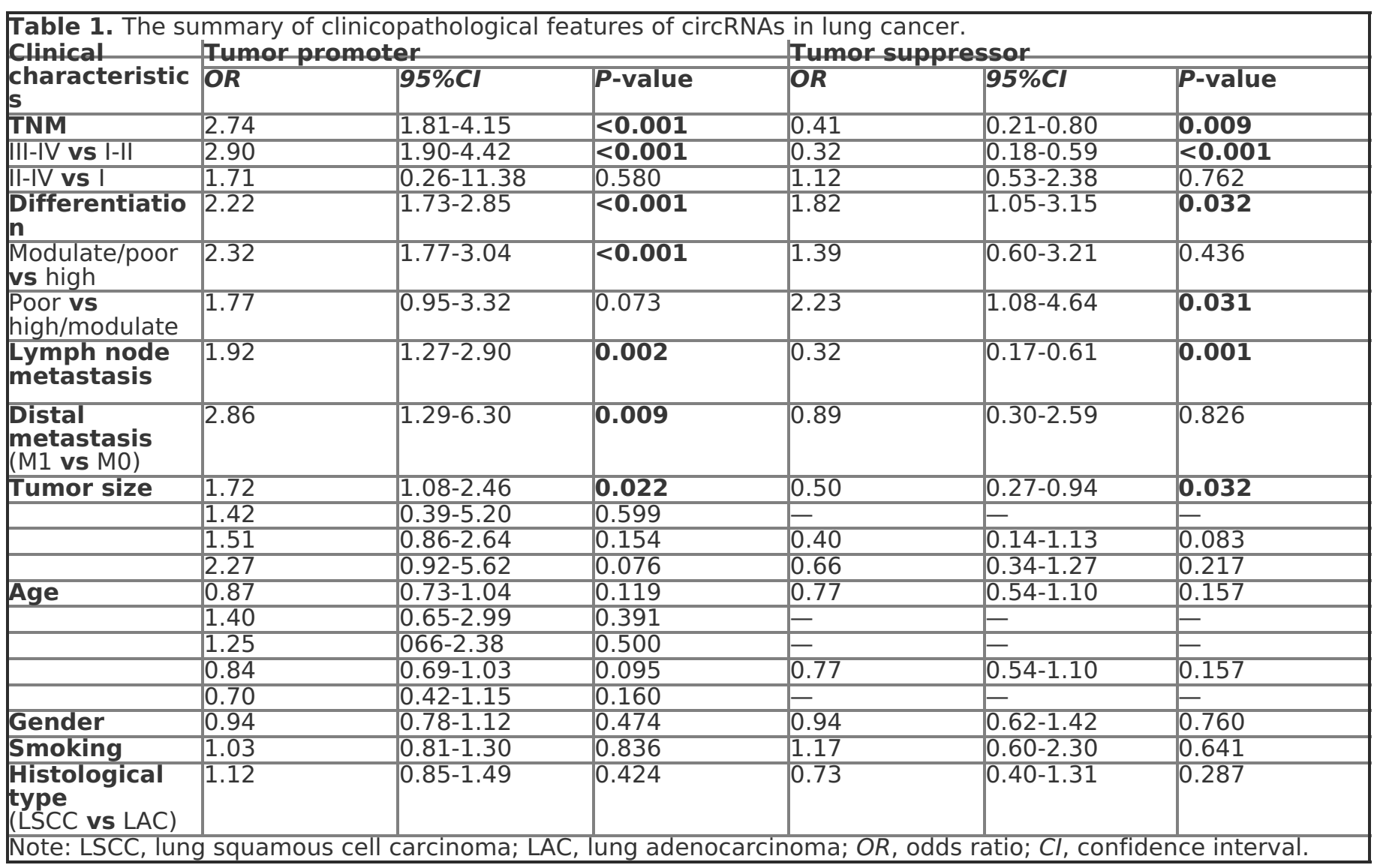




\begin{tabular}{|c|c|c|c|c|c|c|c|c|c|c|}
\hline Study & Year & CircRNA & Cancer & Sample & & Regulati & Sample & Diag & sis power & \\
\hline & & & Type & Case & Control & on & Type & SEN & SPE & AUC \\
\hline \begin{tabular}{|l} 
Xiaoxia \\
Liu \\
[6]
\end{tabular} & 2019 & $\begin{array}{l}\text { hsa_circ_0 } \\
005962\end{array}$ & LAC & 153 & 54 & Up & Plasma & 0.72 & 0.72 & 0.73 \\
\hline $\begin{array}{l}\text { Lijian } \\
\text { Chen } \\
\text { [7] }\end{array}$ & 2019 & $\begin{array}{l}\text { hsa_circ_1 } \\
00146\end{array}$ & NSCLC & 40 & 40 & Up & Tissue & 0.76 & 0.58 & 0.64 \\
\hline $\begin{array}{l}\text { lipeng Lí } \\
\text { [8] }\end{array}$ & 2018 & $\begin{array}{l}\text { hsa_circ_0 } \\
079530^{-0}\end{array}$ & NSCLC & 92 & 92 & Up & Tissue & 0.76 & 0.72 & 0.76 \\
\hline $\begin{array}{l}\text { Liang } \\
\text { Zong } \\
\text { [9] }\end{array}$ & 2018 & $\begin{array}{l}\text { hsa_circ_1 } \\
022 \overline{3} 1\end{array}$ & LAC & 57 & 57 & Up & Tissue & 0.81 & 0.89 & 0.90 \\
\hline $\begin{array}{l}\text { Shaoyan } \\
\text { Zhang } \\
\text { [10] }\end{array}$ & 2018 & $\begin{array}{l}\text { hsa_circ_0 } \\
014 \overline{1} 30^{-}\end{array}$ & NSCLC & 46 & 46 & Up & Tissue & 0.87 & 0.85 & 0.88 \\
\hline $\begin{array}{l}\text { Xiuyuan } \\
\text { Li } \\
\text { [11] }\end{array}$ & 2018 & circ-PVT1 & NSCLC & 45 & 45 & Up & Tissue & 0.83 & 0.68 & 0.80 \\
\hline $\begin{array}{l}\text { Xiuyuan } \\
\text { Li } \\
\text { [11] }\end{array}$ & 2018 & circ-PVT1 & NSCLC & 45 & 45 & Up & Serum & 0.71 & 0.80 & 0.79 \\
\hline $\begin{array}{l}\text { Xiaoli } \\
\text { Zhu } \\
\text { [12] }\end{array}$ & 2017 & $\begin{array}{l}\text { hsa_circ_0 } \\
013958^{-}\end{array}$ & LAC & 49 & 49 & Up & Tissue & 0.76 & 0.80 & 0.82 \\
\hline $\begin{array}{l}\text { Xiaoli } \\
\text { Zhu } \\
\text { [12] }\end{array}$ & 2017 & $\begin{array}{l}\text { hsa_circ_0 } \\
013 \overline{9} 58^{-}\end{array}$ & LAC & 30 & 30 & Up & Plasma & 0.67 & 0.93 & 0.80 \\
\hline $\begin{array}{l}\text { Chunmei } \\
\text { Fan } \\
{[13]}\end{array}$ & 2019 & $\begin{array}{l}\text { Circ- } \\
\text { MAN1A2 }\end{array}$ & LC & 45 & 121 & Up & Serum & 0.51 & 0.79 & 0.65 \\
\hline $\begin{array}{l}\text { Xiaoxia } \\
\text { Liu } \\
\text { [6] }\end{array}$ & 2019 & $\begin{array}{l}\text { hsa_circ_0 } \\
086414\end{array}$ & LAC & 153 & 54 & Down & Plasma & 0.77 & 0.67 & 0.78 \\
\hline $\begin{array}{l}\text { Yanni } \\
\text { Zhang } \\
{[14]}\end{array}$ & 2018 & $\begin{array}{l}\text { Circ- } \\
\text { FOXO3 }\end{array}$ & NSCLC & 45 & 45 & Down & Tissue & 0.8 & 0.73 & 0.78 \\
\hline $\begin{array}{l}\text { Fei Xu } \\
\text { [15] }\end{array}$ & 2018 & $\begin{array}{l}\text { hsa_circ_0 } \\
072309^{-}\end{array}$ & LSCC & 43 & 43 & Down & Tissue & 0.88 & 0.74 & 0.87 \\
\hline $\begin{array}{l}\text { Fei Xu } \\
{[15]}\end{array}$ & 2018 & $\begin{array}{l}\text { hsa circ_0 } \\
006 \overline{1} 14\end{array}$ & LSCC & 43 & 43 & Down & Tissue & 0.86 & 0.72 & 0.82 \\
\hline $\begin{array}{l}\text { Fei Xu } \\
\text { [15] }\end{array}$ & 2018 & $\begin{array}{l}\text { hsa_circ_0 } \\
006460^{-}\end{array}$ & LSCC & 43 & 43 & Down & Tissue & 0.81 & 0.70 & 0.78 \\
\hline $\begin{array}{l}\text { Fei Xu } \\
{[15]}\end{array}$ & 2018 & $\begin{array}{l}\text { hsa_circ_0 } \\
077837^{-}\end{array}$ & LSCC & 43 & 43 & Down & Tissue & 0.86 & 0.81 & 0.86 \\
\hline
\end{tabular}




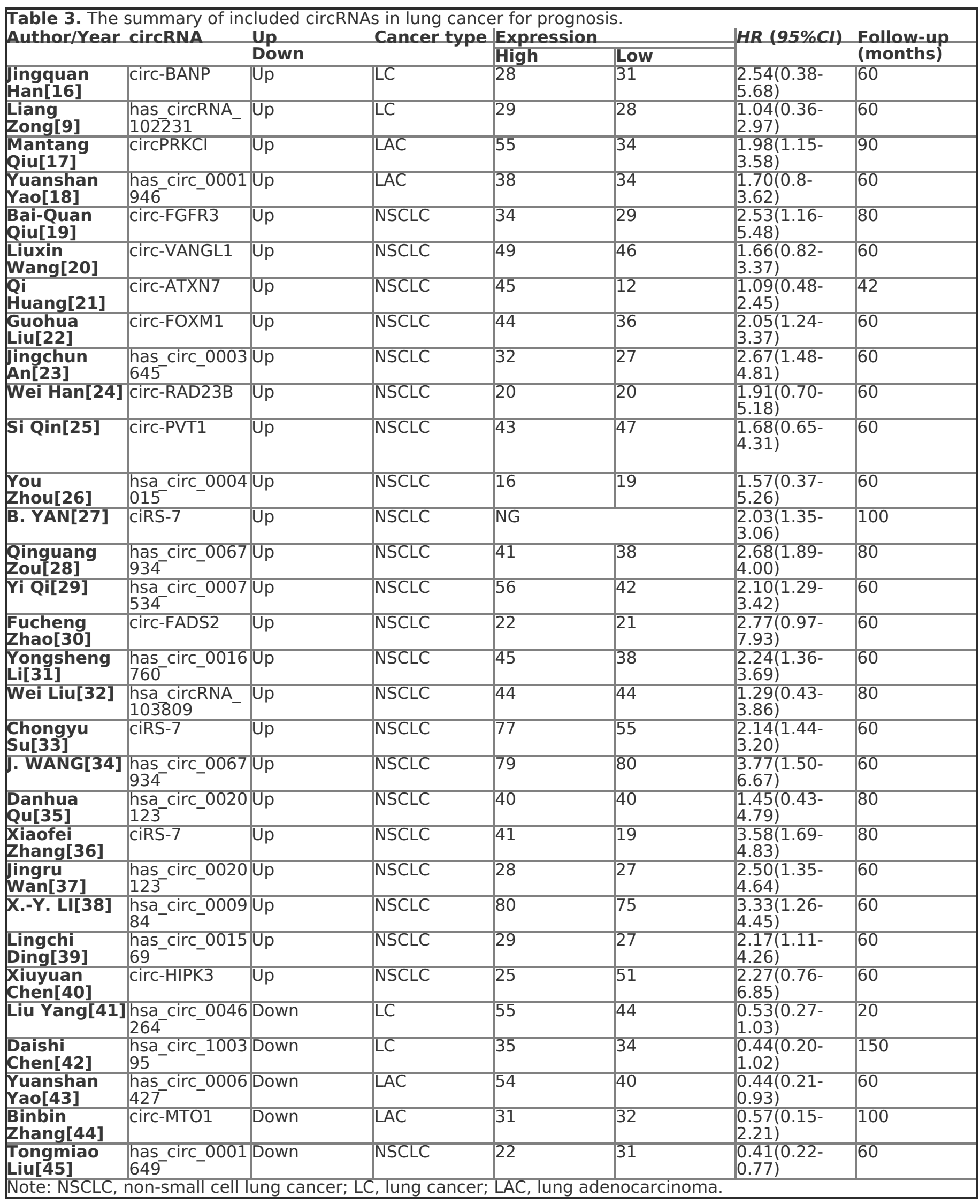




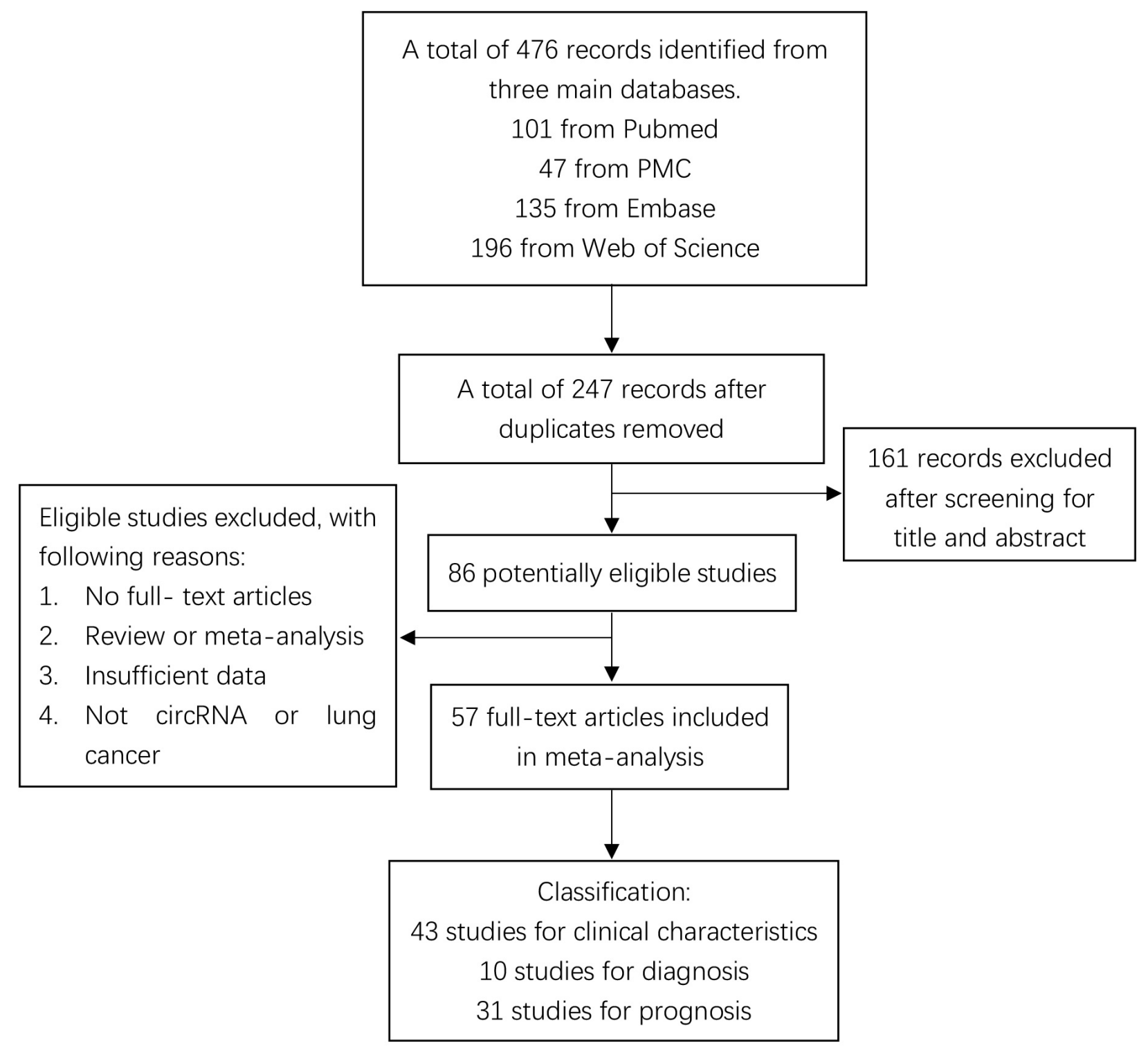

Figure 1

The flowchart showed the algorithm how to perform the exclusion and inclusion of the eligible studies in our meta-analysis.
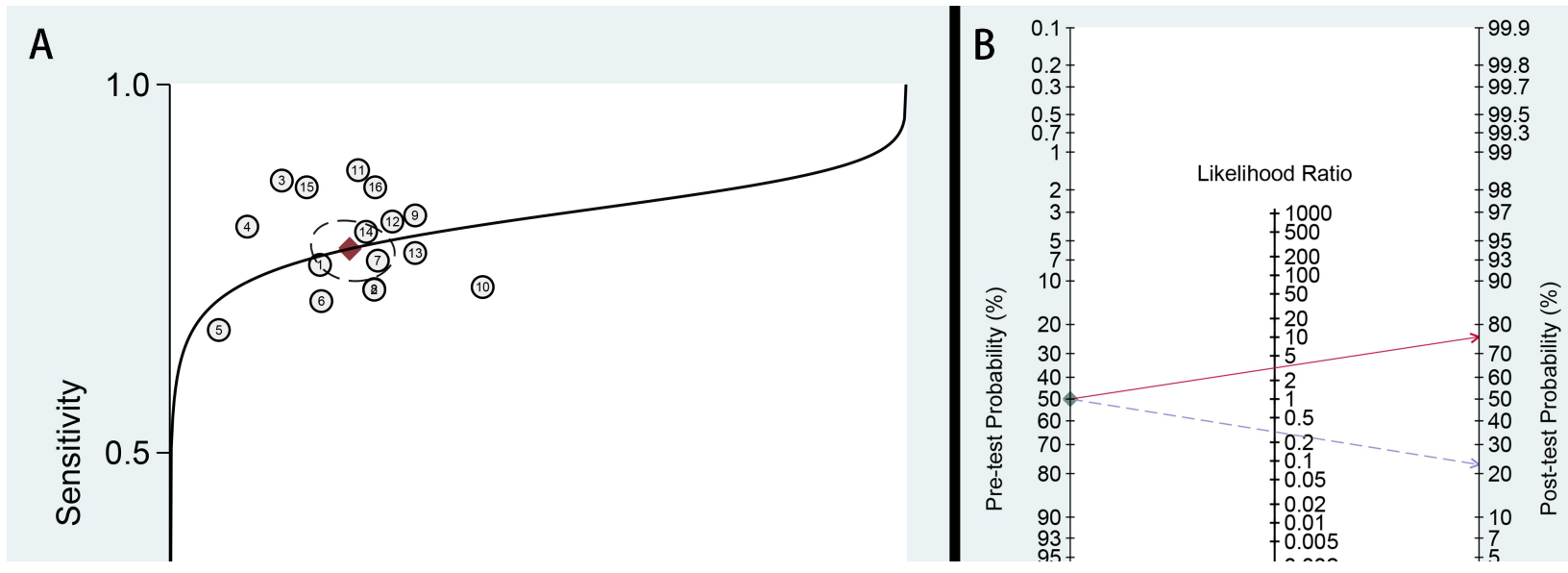


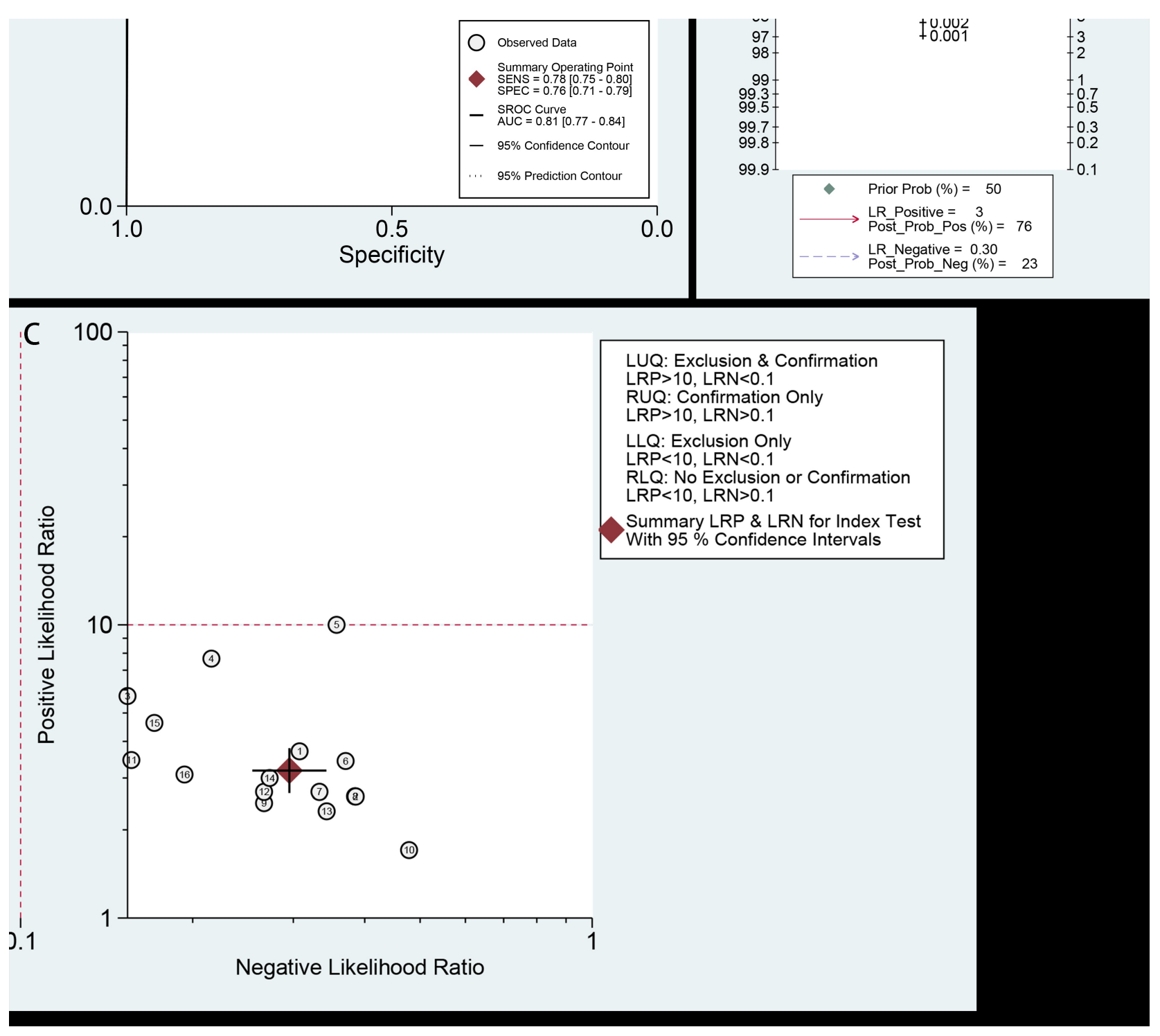

Figure 2

The clinical application of circRNAs in lung cancer. (A) The ability of circRNAs to differentiate patients with lung cancer from normal people was calculated from the Summary receiver operating characteristic (SROC) curve. (B) Fagan's nomogram was shown to estimate the clinical utility of overall circRNAs to differentiate patients with lung cancer from normal people. (C) Likelihood ratio diagram showed the clinical diagnosis value of circRNAs in lung cancer. 

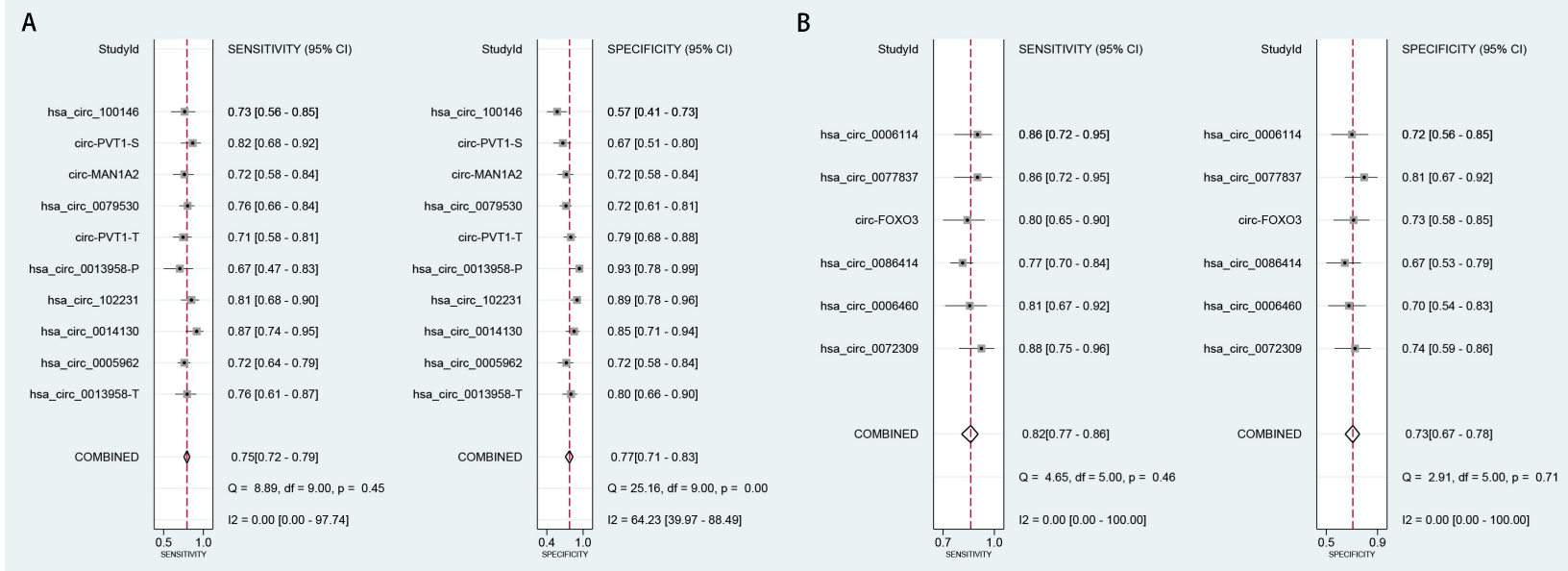

Figure 3

Forest plot by subgroup analysis of diagnosis value in the application of circRNAs in lung cancer. (A) Upregulated circRNAs; (B) Downregulated circRNAs.
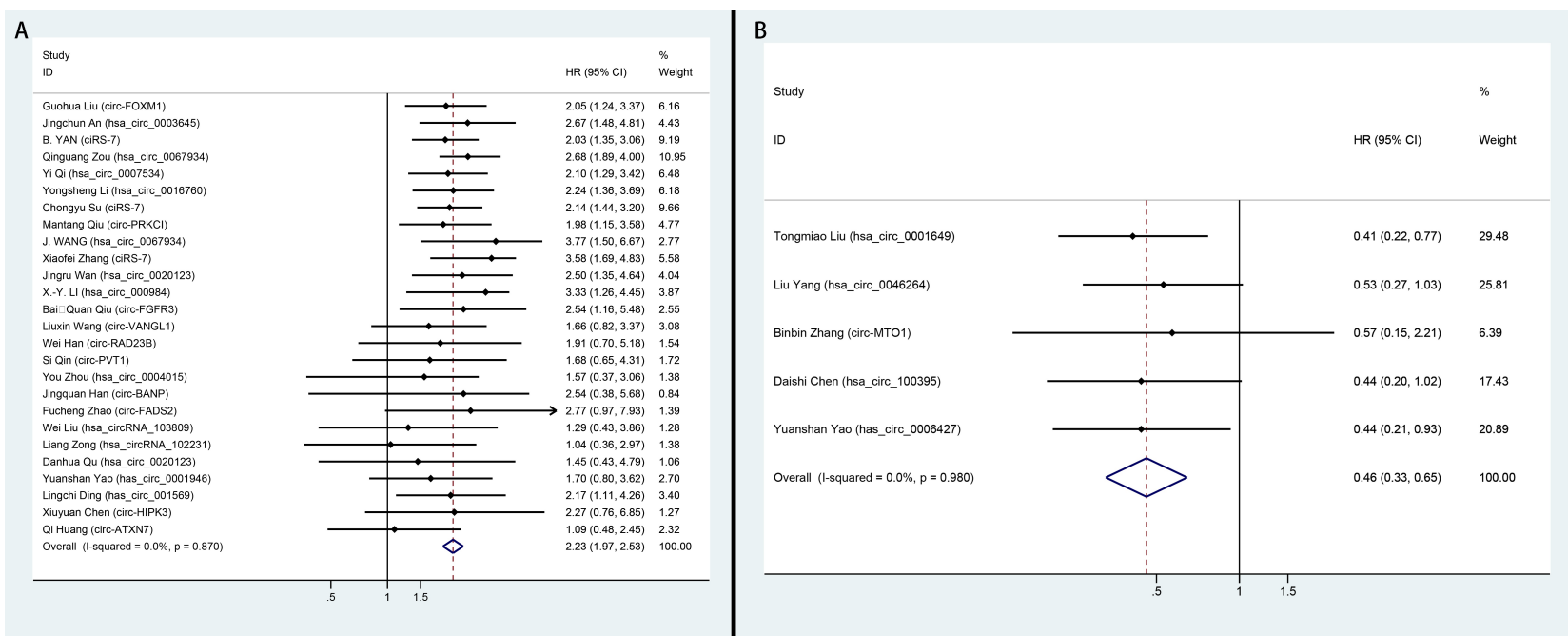

Figure 4

Forest plot of overall survival for prognosis of circRNAs in lung cancer. (A) Upregulated circRNAs; (B) Downregulated circRNAs.

\section{Supplementary Files}

This is a list of supplementary files associated with this preprint. Click to download. supplementary.rar 